\title{
Efficient Cartesian-grid-based modeling of rotationally symmetric bodies
}

\author{
Shyroki, Dzmitry
}

Published in:

I E E E Transactions on Microwave Theory and Techniques

Link to article, DOI:

10.1109/TMTT.2007.897841

Publication date:

2007

Document Version

Publisher's PDF, also known as Version of record

Link back to DTU Orbit

Citation (APA):

Shyroki, D. (2007). Efficient Cartesian-grid-based modeling of rotationally symmetric bodies. I E EE Transactions on Microwave Theory and Techniques, 55(6), 1132-1138.

https://doi.org/10.1109/TMTT.2007.897841

\section{General rights}

Copyright and moral rights for the publications made accessible in the public portal are retained by the authors and/or other copyright owners and it is a condition of accessing publications that users recognise and abide by the legal requirements associated with these rights.

- Users may download and print one copy of any publication from the public portal for the purpose of private study or research.

- You may not further distribute the material or use it for any profit-making activity or commercial gain

- You may freely distribute the URL identifying the publication in the public portal

If you believe that this document breaches copyright please contact us providing details, and we will remove access to the work immediately and investigate your claim. 


\title{
Efficient Cartesian-Grid-Based Modeling of Rotationally Symmetric Bodies
}

\author{
Dzmitry M. Shyroki
}

\begin{abstract}
Axially symmetric waveguides, resonators, and scatterers of arbitrary cross section and anisotropy in the cross section can be modeled rigorously with use of 2-D Cartesian-grid-based codes by means of mere redefinition of material permittivity and permeability profiles. The method is illustrated by the frequencydomain simulations of resonant modes in a circular-cylinder cavity with perfectly conducting walls, a shielded uniaxial anisotropic dielectric cylinder, and an open dielectric sphere for which, after proper implementation of the perfectly matched layer boundary conditions, the radiation quality factor $Q$ is also determined.
\end{abstract}

Index Terms-Body of revolution (BOR), coordinate transformation, finite-difference frequency-domain (FDFD) method, Maxwell equations, perfectly matched layer (PML).

\section{INTRODUCTION}

D IMENSIONALITY reduction is a natural and elegant way to model electromagnetic resonators, waveguides, and scatterers possessing rotational symmetry. It has been used for nearly half a century with integral-equation methods [1], , as well as in the later finite-difference time-domain and finite-element studies-from modeling guided waves in particle accelerators [2, Ch. 12] to ultrashort pulse focusing [3], scattering from axially symmetric targets [4], and cavity mode analysis [5]. The efficiency of these techniques, as compared to straightforward 3-D finite-difference modeling, is pronounced at no accuracy sacrificed. Furthermore, with a "2.5-D" computation workload, the cylindric-grid-based simulators are able to treat systems exhibiting perturbed or discrete rotational symmetry. From the practical view, however, there is one dissatisfactory issue with all such schemes. A conventional approach to reduce dimensionality for rotationally symmetric problems is by performing differentiation with respect to the azimuthal coordinate analytically after casting the Maxwell equations in anholonomic cylindric frame-locally Cartesian - with vector differential operators modified accordingly [6]. The dimensions of the electromagnetic field components remain unchanged under such transformation, but the resulting form of the equations, as opposed to their Cartesian version, is modified. This obviates the need for a separate program implementation of the algorithms based on the Maxwell equations in anholonomic cylindric coordinate system.

Manuscript received November 12, 2006; revised March 13, 2007.

The author is with the Department of Communications, Optics, and Materials, Technical University of Denmark, 2800 Lyngby, Denmark (e-mail: ds@com. dtu.dk).

Digital Object Identifier 10.1109/TMTT.2007.897841
In this paper, a simple way to retain Cartesian-like Maxwell equations and, hence, to use standard Cartesian-grid-based algorithms while actually doing the finite-difference body-of-revolution modeling in cylindrical, spherical, or other rotationally symmetric coordinates, is proposed. We use the generally covariant formulation of Maxwell equations as was propelled, in particular, by Schouten [7] and Post [8] years ago; hence, our scheme involves appropriate transformation of the dielectric permittivity and magnetic permeability together with, where necessary, the electric and magnetic field components instead of modifying the core differential, or finite-difference, equations. It is interesting to note that, in spite of its obvious appeal, this is still a marginal approach in computational electrodynamics, utilized in only a few publications thus far. Thus, Ward and Pendry [9] realized the power of covariant formulations permitting a single "code to handle any coordinate system by adjusting the $\epsilon$ and $\mu$ " in 1996, and implemented this idea in their nonorthogonal logically rectangular finite-difference time-domain code [10], [11]. Covariant 3-D Maxwell equations were used in a recent study [12] on the finite-element modeling of twisted optical fibers in nonorthogonal helical coordinates. Much earlier, in [13] and a series of subsequent papers, covariant transformations were employed to analyze diffraction gratings of complicated profiles in a coordinate system adjusted to the dielectric discontinuity surface. Very recently [14], Maxwell covariance was used for mapping the infinite free space onto the finite computational domain in order to avoid the notorious problem of mesh truncation in the finite-difference computations.

In the following, we derive, after briefly recalling the generally covariant formulation of Maxwell equations, the exact equivalent permittivity and permeability profiles for rotationally symmetric body of arbitrary cross section and anisotropy in the cross section, and for the anisotropic perfectly matched layer (PML) medium used customarily in the finite-difference simulations of radiation and scattering losses. Afterwards, three validating numeric examples are provided via the Yee-mesh-based full-vector 2-D finite-difference frequency-domain calculations of resonant modes. The first example is a canonic circular-cylinder cavity with perfect electric conducting (PEC) walls for which an analytic solution is well known and the finite-difference treatment is not burdened by, e.g., material discontinuities or complicated boundary conditions, thus allowing to focus on how adequate for numeric modeling the proposed equivalent profile method itself is. The second example is a PEC-shielded uniaxially anisotropic dielectric resonator analyzed in [15] with the mode-matching method, and later modeled in [16] with the 
finite-difference tool; with this example, we demonstrate the efficiency of a polarization-sensitive dielectric index averaging at discontinuities on a rectangular finite-difference grid. The third example is an open dielectric sphere, upon which resonant frequencies and $Q$-factors can be calculated analytically [17], [18] and were modeled with the finite-element method in cylindric coordinates [5]; several finite-difference grids to represent the cross section of the sphere are compared and the performance of cylindrical and spherical PMLs to simulate radiation losses is considered.

\section{FORMULATION}

\section{A. Covariant Maxwell Equations}

Here we give a covariant formulation of Maxwell equations in 3-D form most resembling the customary 3-D Cartesian representation. We start from a system [7], [8]

$$
\begin{aligned}
2 \partial_{[\lambda} E_{\nu]} & =-\dot{B}_{\lambda \nu} \\
\partial_{[\kappa} B_{\lambda \nu]} & =0 \\
\partial_{\nu} \mathfrak{H}^{\lambda \nu} & =\dot{\mathfrak{D}}^{\lambda}+\mathfrak{j}^{\lambda} \\
\partial_{\lambda} \mathfrak{D}^{\lambda} & =\rho .
\end{aligned}
$$

Here, the square brackets denote alternation $\cdot[\lambda \nu]=$ $(1 / 2 !)\left(\cdot \cdot_{\lambda \nu}-\cdot_{\nu \lambda}\right)$ and $\cdot_{[\kappa \lambda \nu]}=(1 / 3 !)\left({ }^{\kappa \lambda \nu}+{ }_{\nu \kappa \lambda}+{ }_{\lambda \nu \kappa}-\right.$ $\left.\cdot_{\kappa \nu \lambda}-\cdot_{\lambda \kappa \nu}-\cdot_{\nu \lambda \kappa}\right), E_{\lambda}$ and $B_{\lambda \nu}=-B_{\nu \lambda}$ are the covariant electric vector and magnetic bivector, respectively, coinciding with the electric and magnetic three-vectors $\mathbf{E}$ and $\mathbf{B}$ in a Cartesian frame, $\mathfrak{H}^{\lambda \nu}=-\mathfrak{H}^{\nu \lambda}$ and $\mathfrak{D}^{\lambda}$ are the contravariant magnetic bivector density and the electric vector density of weight +1 corresponding to $\mathbf{H}$ and $\mathbf{D}, \mathfrak{j}^{\lambda}$ and $\rho$ are the electric current and charge densities, and $\kappa, \lambda$, and $\nu=1, \ldots, 3$. With such transformation characteristics assigned to the electromagnetic field quantities, (1) and (2) are known to be form invariant [7], [8], i.e., they do not change their form under arbitrary reversible coordinate transformations. To convert (1) and (2) to the form directly reminiscent of the Maxwell equations in a Cartesian frame, we use the dual equivalents $\tilde{\mathfrak{B}}^{\kappa}=(1 / 2) \tilde{\mathfrak{E}}^{\kappa \lambda \nu} B_{\lambda \nu}$ and $\tilde{H}_{\kappa}=(1 / 2) \tilde{\mathfrak{e}}_{\kappa \lambda \nu} \mathfrak{H}^{\lambda \nu}$, where $\tilde{\mathfrak{E}}^{\kappa \lambda \nu}$ and $\tilde{\mathfrak{e}}_{\kappa \lambda \nu}$ are pseudopermutation (hence, tildes) fields equal to the Levi-Civita symbol in any coordinate system

$$
\begin{aligned}
\tilde{\mathfrak{E}}^{\kappa \lambda \nu} \partial_{\lambda} E_{\nu} & =-\mu^{\kappa \lambda} \dot{\tilde{H}}_{\lambda} \\
\partial_{\kappa} \mu^{\kappa \lambda} \tilde{H}_{\lambda} & =0 \\
\tilde{\mathfrak{E}}^{\kappa \lambda \nu} \partial_{\lambda} \tilde{H}_{\nu} & =\epsilon^{\kappa \lambda} \dot{E}_{\lambda}+\mathfrak{j}^{\kappa} \\
\partial_{\kappa} \epsilon^{\kappa \lambda} E_{\lambda} & =\rho
\end{aligned}
$$

while the constitutive relations are implied being $\tilde{\mathfrak{B}}^{\lambda}=\mu^{\lambda \nu} \tilde{H}_{\nu}$, $\mathfrak{D}^{\lambda}=\epsilon^{\lambda \nu} E_{\nu}$. We leave optical activity, nonlocality, and nonlinearity out of scope here, though these phenomena can be treated on a similar ground insofar as the governing Maxwell field [see (1) and (2)] and the accompanying constitutive relations adhere to the principle of general covariance. The transformation behavior of $\tilde{H}_{\lambda}, \tilde{\mathfrak{B}}^{\lambda}, E_{\lambda}$, and $\mathfrak{D}^{\lambda}$ stipulates that the permittivity $\epsilon^{\lambda \nu}(\mathbf{r})$ and permeability $\mu^{\lambda \nu}(\mathbf{r})$ are contravariant tensor densities of weight +1 so that they are transformed as

$$
\epsilon^{\lambda^{\prime} \nu^{\prime}}\left(\mathbf{r}^{\prime}\right)=\left|\operatorname{det} J_{\lambda}^{\lambda^{\prime}}\right|^{-1} J_{\lambda}^{\lambda^{\prime}} J_{\nu}^{\nu^{\prime}} \epsilon^{\lambda \nu}\left(J_{\lambda^{\prime}}^{\lambda} \mathbf{r}^{\prime}\right)
$$
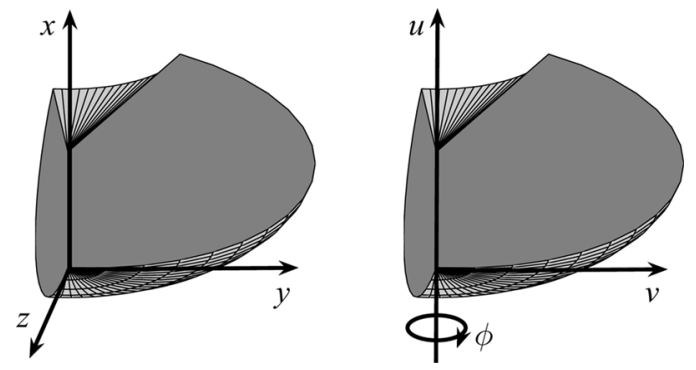

Fig. 1. Cartesian and rotationally symmetric coordinate systems for the given body of revolution. The $x$-axis coincides with rotational symmetry axis of the body; the $\phi=0$ plane corresponds to $z=0, y \geq 0$ in Cartesian coordinates.

where $J_{\lambda}^{\lambda^{\prime}} \equiv \partial_{\lambda} x^{\lambda^{\prime}}$ is the Jacobian transformation matrix, and

$$
\mu^{\lambda^{\prime} \nu^{\prime}}\left(\mathbf{r}^{\prime}\right)=\left|\operatorname{det} J_{\lambda}^{\lambda^{\prime}}\right|^{-1} J_{\lambda}^{\lambda^{\prime}} J_{\nu}^{\nu^{\prime}} \mu^{\lambda \nu}\left(J_{\lambda^{\prime}}^{\lambda} \mathbf{r}^{\prime}\right) .
$$

In this formulation, geometry enters the Maxwell equations (3) and (4) through material fields $\epsilon$ and $\mu$ exclusively, while the form of (3) and (4) is precisely as if they were written in Cartesian components. If, in some chosen coordinate system $\left(x^{1}, x^{2}, x^{3}\right), \epsilon^{\lambda \nu}$ and $\mu^{\lambda \nu}$ happen to be independent of one of the coordinates, say, $x^{3}$, that coordinate can be separated in the usual manner with $\epsilon^{\lambda \nu}=\epsilon^{\lambda \nu}\left(x^{1}, x^{2}\right)$ and $\mu^{\lambda \nu}=\mu^{\lambda \nu}\left(x^{1}, x^{2}\right)$ viewed as the permittivity and permeability profiles of the equivalent dimensionality reduced system in a Cartesian frame. Of course, modeling of rotationally symmetric bodies is only a subset of the possible applications of this technique.

\section{B. Equivalent Body-of-Revolution Profiles}

Depending on the shape of the given body of revolution and its anisotropy, one of many rotationally symmetric coordinate systems can be found advantageous to use. The transformation from orthogonal Cartesian to general rotationally symmetric coordinates, as shown in Fig. 1, is given by [19]

$$
u=u(x, \rho) \quad v=v(x, \rho) \quad \phi=\arctan (z / y)
$$

where $\rho=\sqrt{y^{2}+z^{2}}, u$ and $v$ are the coordinate lines in the $\phi=$ const half-plane-either orthogonal or nonorthogonal, analytically defined, or automatically generated boundaryfitted ones. The corresponding transformation matrix for contravariant components reads

$$
J_{\lambda}^{\lambda^{\prime}}=\left[\begin{array}{ccc}
u_{x}^{\prime} & u_{\rho}^{\prime} \cos \phi & u_{\rho}^{\prime} \sin \phi \\
v_{x}^{\prime} & v_{\rho}^{\prime} \cos \phi & v_{\rho}^{\prime} \sin \phi \\
0 & -\rho^{-1} \sin \phi & \rho^{-1} \cos \phi
\end{array}\right] .
$$

Before substituting this in (5) and (6), we factor out the $\phi$ dependence $[J]=\left[J_{0}\right]\left[R_{x}\right]^{\mathrm{T}}$, where ${ }^{\mathrm{T}}$ denotes transposition, $\left[R_{x}\right]=$ $\left[R_{x}(\phi)\right]$ is the matrix of rotation around $x$, and

$$
\left[J_{0}\right]=\left[\begin{array}{ccc}
u_{x}^{\prime} & u_{\rho}^{\prime} & 0 \\
v_{x}^{\prime} & v_{\rho}^{\prime} & 0 \\
0 & 0 & \rho^{-1}
\end{array}\right]
$$

is the Jacobian at $\phi=0$. For a body of arbitrary cross section and anisotropy in the cross section, if only invariant under rotations around the $x$ axis, i.e., $\left[\epsilon_{\mathrm{C}}\right]=\left[R_{x}\right]\left[\epsilon_{0}\right]\left[R_{x}\right]^{\mathrm{T}}$, where $\left[\epsilon_{0}\right]$ 
is $\left[\epsilon_{\mathrm{C}}\right]$ at $\phi=0$, and similarly for $\mu_{\mathrm{C}}$, one finally gets after applying the $R_{x}^{\mathrm{T}} R_{x}=1$ identities

$$
\begin{aligned}
{[\epsilon] } & =\left|\operatorname{det} J_{0}\right|^{-1}\left[J_{0}\right]\left[\epsilon_{0}\right]\left[J_{0}\right]^{\mathrm{T}} \\
{[\mu] } & =\left|\operatorname{det} J_{0}\right|^{-1}\left[J_{0}\right]\left[\mu_{0}\right]\left[J_{0}\right]^{\mathrm{T}} .
\end{aligned}
$$

Since such transformed permittivity and permeability do not depend on the $\phi$ coordinate, the standard separation of variables in (3) and (4) is in order with $\mathrm{e}^{\mathrm{i} 2 \pi m \phi}$ factored out. At the same time, it should be emphasized that the form of the Maxwell equations (3) and (4) remains exactly Cartesian like, whatever the $u$ and $v$ coordinates and the associated "physical" grid in the body cross section is preferred to be employed.

In the specific case of cylindric coordinates, one has $u=$ $x$ and $v=\rho$, hence, $\left[J_{0}\right]=\operatorname{diag}\left\{1,1, \rho^{-1}\right\}$ in the above formulas. For the diagonal-anisotropic media, i.e., if $\left[\epsilon_{0}\right]=$ $\operatorname{diag}\left\{\epsilon_{\mathrm{C} x}, \epsilon_{\mathrm{C} \rho}, \epsilon_{\mathrm{C} \phi}\right\}$, as measured in a locally Cartesian reference frame, one thus obtains

$$
[\epsilon]=\operatorname{diag}\left\{\epsilon_{\mathrm{C} x} \rho, \epsilon_{\mathrm{C} \rho} \rho, \epsilon_{\mathrm{C} \phi} \rho^{-1}\right\}
$$

and, similarly, for $[\mu]$. In the case of a spherical coordinate system (we use it alongside with the cylindric one in Section III-C) with $u=\sqrt{x^{2}+\rho^{2}} \equiv r$ and $v=\arctan (\rho / x) \equiv \theta$, one gets for the equivalent profiles of isotropic media

$$
[\epsilon]=\epsilon_{\mathrm{C}} \operatorname{diag}\left\{r^{2} \sin \theta, \sin \theta, \sin ^{-1} \theta\right\}
$$

and similarly for $[\mu]$. The equivalent permittivity profile for the diagonal-anisotropic media $\left[\epsilon_{0}\right]=\operatorname{diag}\left\{\epsilon_{\mathrm{Cr} r}, \epsilon_{\mathrm{C} \theta}, \epsilon_{\mathrm{C} \phi}\right\}$ is now represented by a nondiagonal matrix.

\section{PMLs}

The primary way to simulate radiation losses with the finite-difference or finite-element tools is by introducing the PML boundaries [2, Ch. 7]. We construct the "uniaxial" PMLs below by appropriate complex coordinate stretching [20], [21]. For cylindrical PMLs, for example, we employ the scaling from $\hat{\rho}$ to $\rho$ via

$$
\hat{\rho}=\rho_{0}+\int_{\rho_{0}}^{\rho} s_{\rho} \mathrm{d} \rho
$$

with the complex parameter $s_{\rho}=J_{\rho}^{\hat{\rho}}$ often chosen as

$$
s_{\rho}=1+\mathrm{i} \sigma_{\max }\left(\frac{\rho-\rho_{0}}{w_{\rho}}\right)^{p}, \quad \text { for } \rho_{0}<\rho \leq \rho_{0}+w_{\rho}
$$

and the similar scaling $\hat{x}=\hat{x}(x)$ with

$$
s_{x}=1+\mathrm{i} \sigma_{\max }\left(\frac{|x|-x_{0}}{w_{x}}\right)^{p}, \quad \text { for } x_{0}<|x| \leq x_{0}+w_{x}
$$

where $\sigma_{\max }$ is the dimensionless frequency-dependent maximum PML conductivity, $x= \pm x_{0}$ and $\rho=\rho_{0}$ are the PML interfaces, $w_{x}$ and $w_{\rho}$ are the PML widths in the $x$ - and $\rho$-directions, $p$ is the conductivity polynomial profile order, and the plus sign before the imaginary parts implies the $\exp (-\mathrm{i} \omega t)$ time dependence of the fields in the frequency domain.

The equivalent permittivity (12) further transformed with $[J]=\operatorname{diag}\left\{s_{x}^{-1}, s_{\rho}^{-1}, 1\right\}$ within the PML regions becomes

$$
[\epsilon]=\operatorname{diag}\left\{\epsilon_{\mathrm{C} x} \frac{s_{\rho} \hat{\rho}}{s_{x}}, \epsilon_{\mathrm{C} \rho} \frac{s_{x} \hat{\rho}}{s_{\rho}}, \epsilon_{\mathrm{C} \phi} \frac{s_{x} s_{\rho}}{\hat{\rho}}\right\}
$$

and, similarly, for $[\mu]$. For the spherical PMLs imposed on the equivalent material profiles (13), one gets

$$
[\epsilon]=\epsilon_{\mathrm{C}} \operatorname{diag}\left\{\frac{\hat{r}^{2} \sin \theta}{s_{r}}, s_{r} \sin \theta, \frac{s_{r}}{\sin \theta}\right\}
$$

with $\hat{r}$ and $s_{r}$ defined similarly to (14) and (15). Extensions to the more complicated cases are clear.

\section{VALIDATION}

For the three numeric examples here, we specify the equivalent profiles of the resonators according to (12) or (13) and discretize them on a 2-D projected Yee grid with appropriate boundary conditions. The discretized version of Maxwell equations (3) and (4), or rather the reduced eigenproblem in the frequency squared and in $\left\{H_{u}, H_{v}\right\}^{T}$ or $\left\{E_{u}, E_{v}\right\}^{T}$, is then solved iterationally via ARPACK [22] based eigs function in MATLAB. ${ }^{1}$ Although the time-domain simulations are certainly possible as well, the frequency domain is naturally preferred in finding resonant modes here. The choice for the finite-difference, as opposed to the integral-equation methods, is dictated by the fact that even for the homogeneous bodies, the equivalent profiles are continuously inhomogeneous in the radial direction; and this choice is supported, on the physical part, by the high efficiency of the finite-different solvers in dealing with the often complicated microstructured profiles of the current and the prospective real-world devices.

\section{A. Empty Cylinder Cavity With PEC Walls}

This canonic example [23], unobscured by any complexities such as nontrivial boundary conditions or material profile discontinuities, permits to scrutinize the core of the method-the equivalent-profile formulation itself, as implemented for the finite-difference modeling. We chose a cylinder with the height-to-radius ratio equal to the golden ratio $\Phi=1.680$ and simulated, on the $X \times R=1.680 a \times a$ domain, several of the lowest resonant modes with precalculated frequencies $f_{0}=2.6758$ (for the $\mathrm{TE}_{111}$ mode), $4.2956\left(\mathrm{TE}_{011}\right), 5.6740$ $\left(\mathrm{TE}_{121}\right), 2.4048\left(\mathrm{TM}_{010}\right), 3.0908\left(\mathrm{TM}_{011}\right)$, and 5.4904 $\left(\mathrm{TM}_{211}\right)$, all in the $c /(2 \pi a)$ units. Convergence curves for these modes are plotted in Fig. 2, where all the modes show perfect second-order behavior, while the grid resolution increases by more than an order of magnitude. The higher order modes are found with systematically lower accuracy at a given resolution because of their more complicated oscillatory electric and magnetic field profiles (see Fig. 3). Overall, such convergence behavior, together with low absolute values for the errors, is very similar to the results obtained for a straight rectangular resonator with the same finite-difference code after

${ }^{1}$ [Online]. Available: www.mathworks.com 


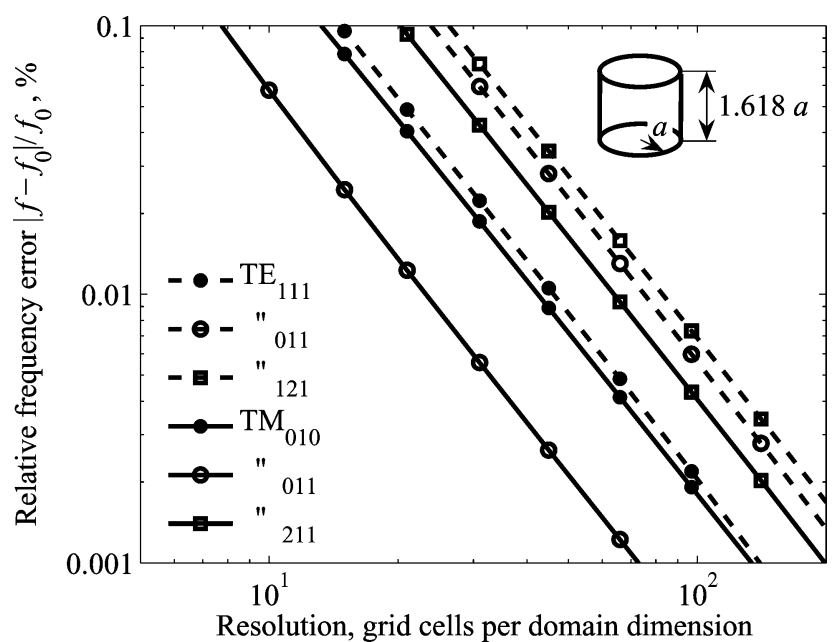

Fig. 2. Convergence of the eigenfrequencies with resolution for some of the lowest TE and TM modes in the air-filled circular-cylinder cavity with perfectly conducting walls.

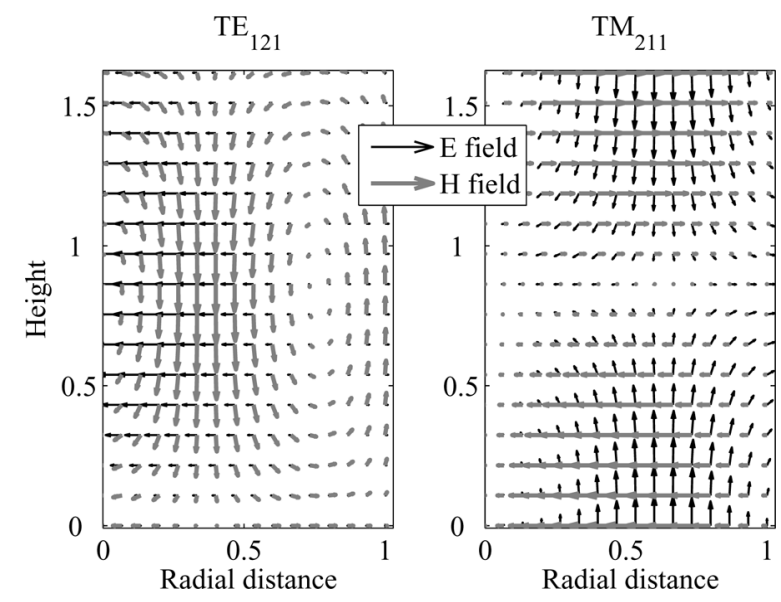

Fig. 3. Axial half cross sections of the electric and magnetic fields, $\pi / 2$ shifted in $\phi$ relative to each other, of the $\mathrm{TE}_{121}$ and $\mathrm{TM}_{211}$ modes in the same resonator as in Fig. 2.

we put $\epsilon=1$ and $\mu=1$, indicating that the equivalent-profile technique itself is not detrimental to the finite-difference modeling accuracy.

\section{B. Shielded Anisotropic Dielectric Resonator}

In this example, we benchmark our method against the rigorous mode-matching results of [15] for the resonant modes in a PEC-shielded uniaxially anisotropic dielectric cylinder resonator, reproduced with good accuracy in [16] employing, in anholonomic cylindric coordinates, the finite difference with simultaneous Chebyshev acceleration method. The diameter and height of the cavity shield are 15.6 and $13.0 \mathrm{~mm}$, respectively, the diameter and the height of the sapphire cylinder centered in the cavity are $10.0 \mathrm{~mm}$ each, and its permittivity $\epsilon=\operatorname{diag}\{11.478,9.389,9.389\}$ so that the anisotropy axis points along the axial or $x$-direction. The dielectric supports of $\epsilon=1.031$ were also included as in [15] and [16]. The convergence graphs for the two lowest hybrid modes $E H_{11 \delta}$ and

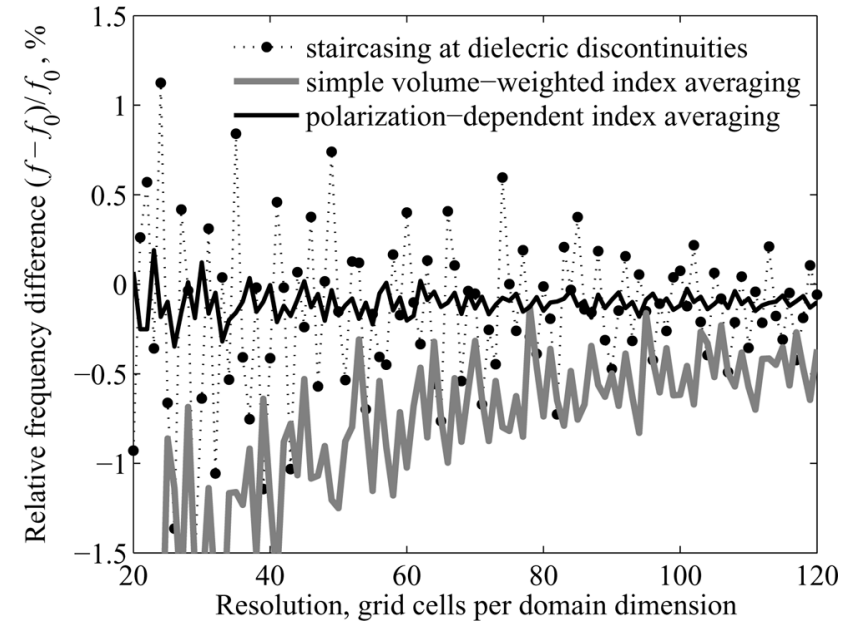

Fig. 4. Convergence for the $E H_{11 \delta}$-mode resonant frequency $f$ to its exact value $f_{0}=8.827 \mathrm{GHz}$ in the PEC-shielded uniaxially anisotropic high-indexcontrast dielectric resonator as in [15] and [16].

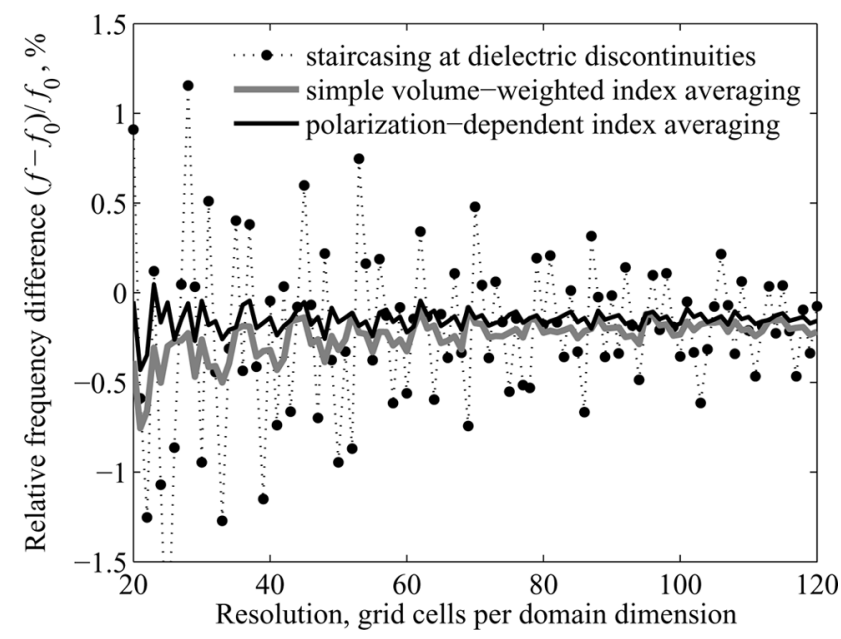

Fig. 5. Convergence for the $H E_{11 \delta}$-mode resonant frequency $f$ to its exact value $f_{0}=9.121 \mathrm{GHz}$ in the same PEC-shielded uniaxially anisotropic resonator as in Fig. 4.

$H E_{11 \delta}$ are plotted in Figs. 4 and 5; the electric and magnetic field profiles of these modes shown in Fig. 6 can be compared to Figs. 3 and 4 in [16].

A common problem with the finite-difference grids, including the rectangular staggered Yee-type grid we use here, is how to represent material discontinuities running along the curved surfaces and curved lines on that grid. Defining the permittivity in a grid cell containing discontinuity as that at the cell center-the staircasing - appears too rough in many cases [24]. Two options follow: either $\epsilon$ and $\mu$ should be averaged carefully over the grid cells crossed by the discontinuities, or adaptive coordinates should be chosen such that the coordinate surfaces follow the material interfaces to avoid the very necessity for the averaging. Now we consider the permittivity averaging approach, leaving the adaptive coordinate transformations until Section III-C.

In Figs. 4 and 5, one can compare the results obtained with the volume-weighted averaging $\epsilon_{\text {eff }}=\langle\epsilon\rangle=(1 / \Omega) \int \epsilon \mathrm{d} \mathbf{r}$ of the 


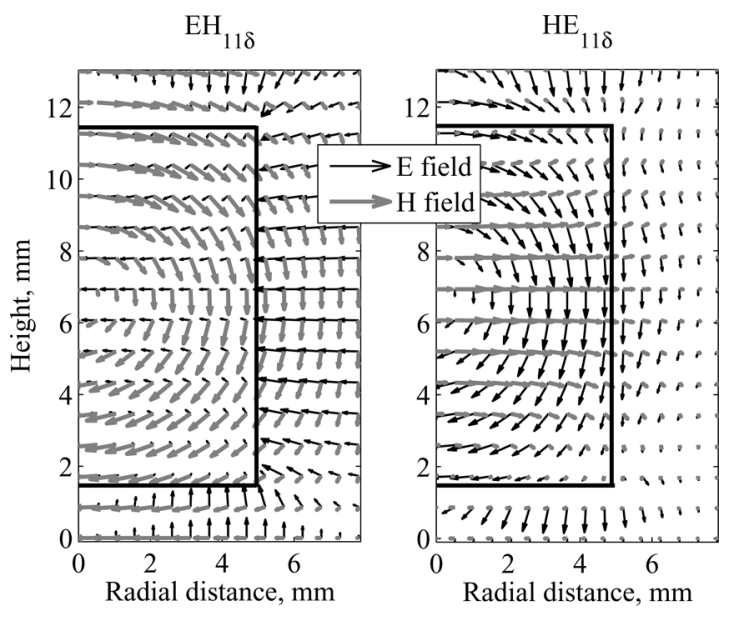

Fig. 6. Axial half cross sections $(\pi / 2$ shifted in $\phi)$ of electric and magnetic fields of the $E H_{11 \delta}$ and $H E_{11 \delta}$ modes for the same resonator as in Figs. 4 and 5 , calculated with a resolution of 80 grid cells per each dimension.

permittivity, as proposed in [25], and supported in the authoritative book [2, Sec. 10.6.3] against the polarization-dependent homogenization [26, Sec. 14.5.2]

$$
\epsilon_{\mathrm{eff}}=\epsilon_{\|}(1-\mathbf{n} \otimes \mathbf{n})+\epsilon_{\perp} \mathbf{n} \otimes \mathbf{n}
$$

for grid cells small enough to assume the discontinuity surface is the plane within each cell, $\mathbf{n}$ being the unit vector normal to that plane; the so-called Wiener limits are $\epsilon_{\mid}=\langle\epsilon\rangle$ and $\epsilon_{\perp}=\left\langle\epsilon^{-1}\right\rangle^{-1}$. (In fact, a more general formula [27] to account for material anisotropy is in order. Furthermore, the off-diagonal components of (19) were ignored in our computations. These details, however, are not pertinent to this discussion.) The systematic underestimation of the eigenfrequencies calculated with volume-weighted averaging, like that observed in [28]-[30] for the TM modes in 2-D photonic crystals or in [31, Sec. 3.3.1] for the hybrid modes in microstructured fibers, is due to $\epsilon_{1} \geq \epsilon_{\perp}$, which can be checked via

$$
\frac{\epsilon_{\|}}{\epsilon_{\perp}}=\frac{1}{\Omega} \int \epsilon \mathrm{d} \mathbf{r} \cdot \frac{1}{\Omega} \int \epsilon^{-1} \mathrm{~d} \mathbf{r} \geq \frac{1}{\Omega^{2}} \iint \epsilon \cdot \epsilon^{-1} \mathrm{~d} \mathbf{r} \mathrm{d} \mathbf{r}=1
$$

valid for any nonnegative function $\epsilon(\mathbf{r})$. The inadequacy of the simplistic volume-weighted averaging is especially well seen for the modes with an electric field vector directed predominantly normally to material interfaces, as for the $E H_{11 \delta}$ mode in our case. Taking $\epsilon_{\perp}$ together with $\epsilon_{\mid}$into account in the finite-difference computations, as prescribed by (19), pointed out in [28] (see also [29]), and implemented in a widely used planewave modeling package MPB [32], improves the accuracy at no serious computation cost.

\section{Open Dielectric Sphere}

The resonant frequencies (free oscillations) and the radiation losses of an isolated sphere can be calculated by employing cylindric functions in the known manner [17], [18], [33], providing an etalon to test our equivalent-profile finite-difference

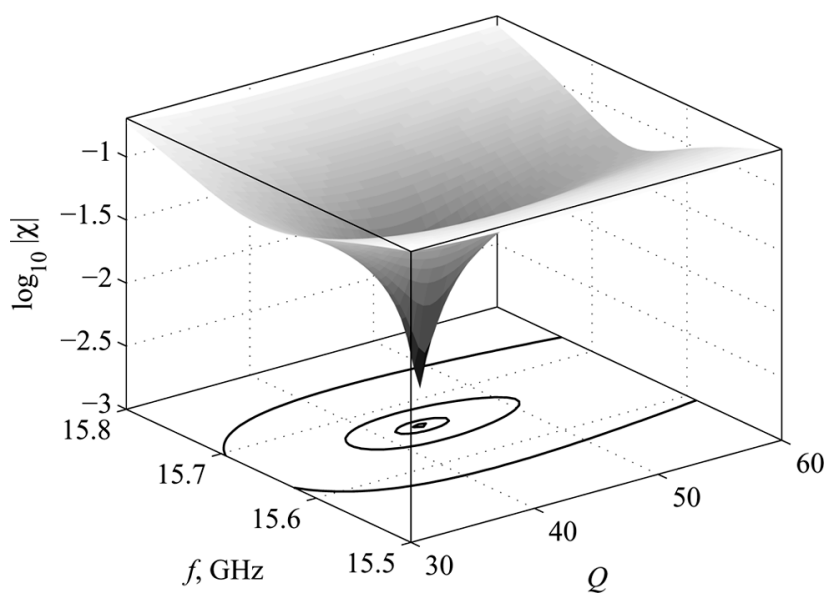

Fig. 7. Localization of the $\mathrm{TE}_{101}$ mode for open dielectric sphere of permittivity $\epsilon=36$ and radius $a=1.56 \mathrm{~mm}$, as in [18], in the frequency $(f)$ versus quality factor $(Q)$ coordinates.

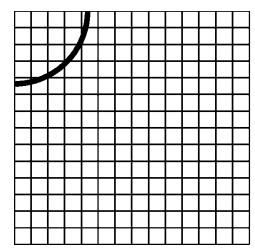

(a)

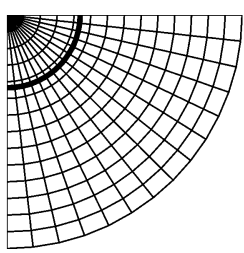

(b)

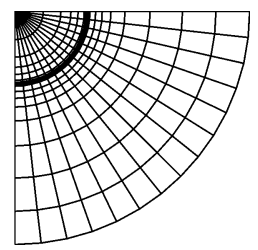

(c)
Fig. 8. Three different "physical" grids that we use. (a) Rectangular. (b) Polar. (c) Polar radially transformed to better represent the dielectric discontinuity.

method with the domain boundaries truncated by PMLs. Consider, for example, the TE modes that correspond to the (necessarily complex) zeros of

$$
\chi=\sqrt{\epsilon} \frac{J_{n-1 / 2}\left(\sqrt{\epsilon} k_{0} a\right)}{J_{n+1 / 2}\left(\sqrt{\epsilon} k_{0} a\right)}-\frac{H_{n-1 / 2}^{(2)}\left(k_{0} a\right)}{H_{n+1 / 2}^{(2)}\left(k_{0} a\right)}
$$

where $J_{n}$ and $H_{n}^{(2)}$ are the first Bessel and the second-kind Hankel functions of the order $n$ and the complex wavenumber $k_{0}=\omega c^{-1}=2 \pi f c^{-1}(1-\mathrm{i} /(2 Q))$. We simulated the $\mathrm{TE}_{101}$ mode for the sphere of the dielectric permittivity $\epsilon=36$ and the radius $a=1.56 \mathrm{~mm}$, as in [18]. It must be mentioned that this mode, and not only this one, was inaccurately located numerically in [18] in the $(f, Q)$-plane. In Fig. 7, a logarithm of $\chi$ is plotted in the vicinity of the $\mathrm{TE}_{101}$-mode minimum. The location of the minimum found with the FindMinimum function in Mathematica ${ }^{2}$ gives $f_{0}=15.661 \mathrm{GHz}$ and $Q_{0}=43.17$, the values supported also by brute-force 3 -D time-domain simulations [34]. The order-of-magnitude difference in the slopes along the $Q$ and $f$ coordinates justifies lower accuracy in determining the $Q$.

The use of a rectangular grid, as in Fig. 8(a), together with the polarization-sensitive averaging of permittivity at the surface of dielectric discontinuity produce pretty good second-order convergence of the simulated results for both $f$ and $Q$ with the $Q$ errors being an order of magnitude higher at a fixed resolution

\footnotetext{
${ }^{2}$ [Online]. Available: www.wolfram.com
} 


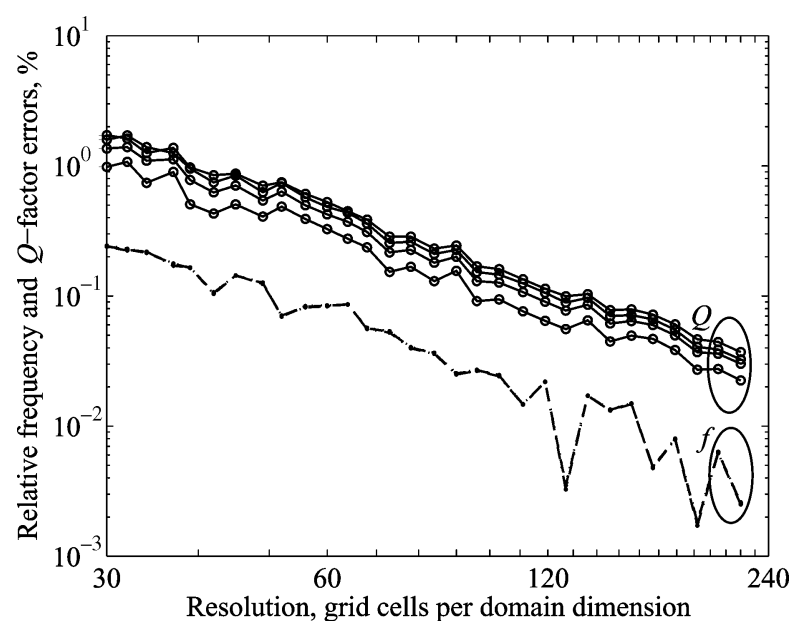

Fig. 9. Convergence for $f$ and $Q$ of the $\mathrm{TE}_{101}$ mode calculated on the $6 \times$ $6 \mathrm{~mm}^{2}$ domain. The curves in each family are obtained with varying the PML parameters; polarization-dependent averaging of permittivity is applied.

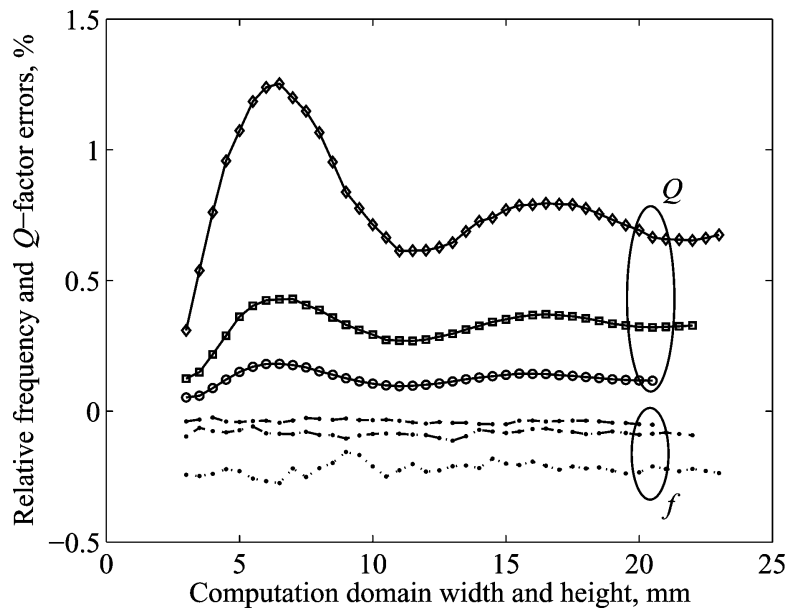

Fig. 10. Sensitivity of $f$ and $Q$ to the computation domain width and height (increasing proportionally) for the resolutions of six (less accurate results in both $f$ and $Q$ ), ten, and (best accurate) 14 grid cells per $1 \mathrm{~mm}$.

(Fig. 9). Simulations were done on the $6 \times 6 \mathrm{~mm}^{2}$ domain representing a quarter of the sphere, with the PEC and perfect magnetic conducting (PMC) boundary conditions on the axis and in the $x=0$ plane, respectively, according to the mode symmetry. The 1-mm-wide PMLs were placed within the domain at the two outer boundaries, and a quadratic conductivity profile was specified with the maximum conductivity corresponding to the continuous-space reflection coefficient $\mathcal{R}=\mathrm{e}^{-10}$. Changing the reflection coefficient to $\mathcal{R}=\mathrm{e}^{-20}$, the width of the PMLs to $w=2 \mathrm{~mm}$, and the polynomial order to $p=4$ does not alter the overall convergence behavior for $Q$, as seen from this same figure, and exposes virtually no effect on the $f$ values. Fig. 10 indicates that, in coarse grid simulations, the $Q$ values are sensitive to the domain width, exhibiting a slowly converging oscillatory behavior. Not so for sufficiently fine grids, however; in that case, one can place PMLs quite close to the simulated object.

A natural alternative to calculations on the physically rectangular grid in this case is to use a polar grid, as in Fig. 8(b), with the equivalent permittivity and permeability profiles as given by

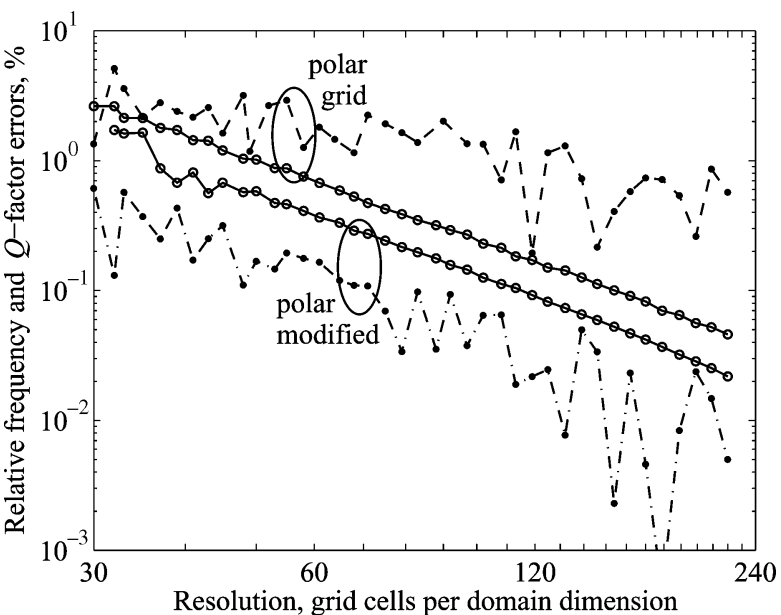

Fig. 11. Convergence for $f$ and $Q$ (compared with Fig. 9) calculated on physically polar grids - unmodified and radially modified, as in Fig. 8, extending for $6 \mathrm{~mm}$ radially. No permittivity averaging applied.

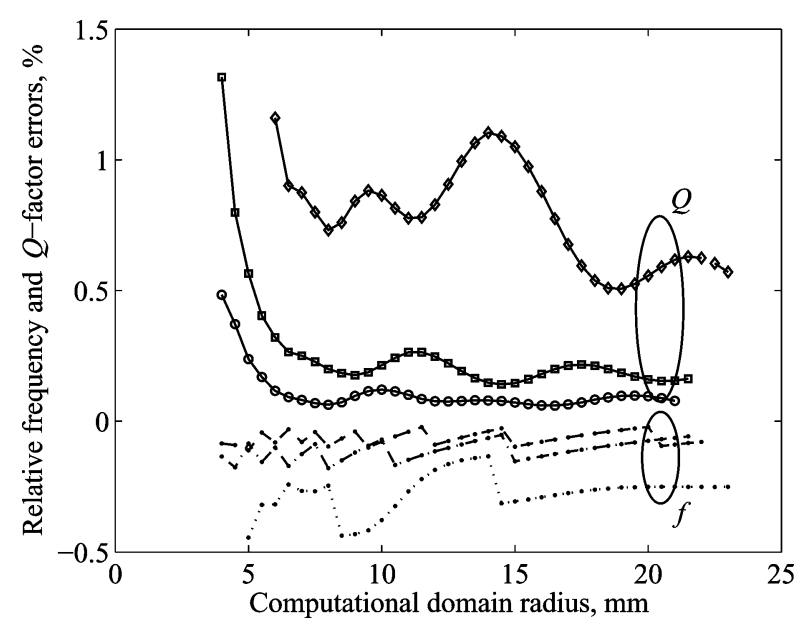

Fig. 12. Sensitivity of $f$ and $Q$ to the domain radius at the same radial resolutions as in Fig. 10. The angular resolution is 30 grid cells per $\pi / 2$ for all the curves.

(13); or polar modified as in Fig. 8(c) to increase the density of grid nodes near dielectric discontinuity, e.g., via

$$
r^{\prime}=r+s \delta\left[1+\frac{2}{\pi} \arctan \left(\frac{\pi}{2} \frac{r-a}{\delta}\right)\right]
$$

with equivalent profiles modified accordingly. Our results for the $f$ and $Q$ convergence and sensitivity to the domain radius (Figs. 11 and 12) were obtained with $s=63$ and $\delta=a / 100$ when the radial transformation was applied. Noteworthy is that we neither performed the permittivity averaging, nor cared to place the discretized coordinate surfaces somehow specifically with respect to material boundaries, but the accuracy achieved on the modified polar grid is comparable to that in Figs. 9 and 10 . Our point, however, is demonstrating not the superiority of adaptive meshing here, but the ease of swapping between different "physical" grids, while using one and the same "logically Cartesian" solver with different equivalent permittivity and permeability profiles. 


\section{CONCLUSION}

The equivalent-profile expressions (10) and (11) for an anisotropic inhomogeneous body of revolution have been derived for further use with the logically Cartesian-grid-based solvers. The following three reasons make our approach appeal.

1) Its computational efficiency is comparable to that of other body-of-revolution techniques which make use of rotational symmetry of the structure by casting Maxwell equations in anholonomic cylindric coordinates, but an arbitrary boundary-fitted grid in the body cross section is acceptable with our approach.

2) Its ease of program implementation is unsurpassed: actually, no or virtually no modifications to the widely available time- or frequency-domain Cartesian-grid-based Maxwell solvers are required, except the adjustments to the domain boundary conditions where necessary.

3) Its conceptual underpinning - the general covariance of Maxwell equations - has apparent applications beyond the case of rotational symmetry considered here. For example, homogeneous twists can be treated in a similar way by the separation of variables in the nonorthogonal helical coordinates.

\section{REFERENCES}

[1] M. G. Andreasen, "Scattering from bodies of revolution," IEEE Trans. Antennas Propag., vol. AP-13, no. 3, pp. 303-310, Mar. 1965.

[2] A. Taflove and S. C. Hagness, Computational Electrodynamics: The Finite-Difference Time-Domain Method, 2nd ed. Boston, MA: Artech House, 2000.

[3] D. B. Davidson and R. W. Ziolkowski, "Body-of-revolution finite-difference time-domain modeling of space-time focusing by a three-dimensional lens," J. Opt. Soc. Amer. A, Opt. Image Sci., vol. 11, pp. $1471-1490,1994$.

[4] A. D. Greenwood and J.-M. Jin, "A novel efficient algorithm for scattering from a complex BOR using mixed finite elements and cylindrical PML," IEEE Trans. Antennas Propag., vol. 47, no. 4, pp. 620-629, Apr. 1999.

[5] O. Chinellato, P. Arbenz, M. Streiff, and A. Witzig, "Computation of optical modes in axisymmetric open cavity resonators," Future Gen. Comput. Syst., vol. 21, pp. 1263-1274, 2005.

[6] D. V. Redžić, "The operator $\nabla$ in orthogonal curvilinear coordinates," Eur. J. Phys., vol. 22, pp. 595-599, 2001.

[7] J. A. Schouten, Tensor Analysis for Physicists. Oxford, U.K.: Clarendon, 1951, (reprinted by Dover, 1989).

[8] E. J. Post, Formal Structure of Electromagnetics. Amsterdam, The Netherlands: North-Holland, 1962, (reprinted by Dover, 1997).

[9] A. J. Ward and J. B. Pendry, "Refraction and geometry in Maxwell's equations," J. Modern Opt., vol. 43, pp. 773-793, 1996.

[10] A. J. Ward and J. B. Pendry, "Calculating photonics Green's functions using a nonorthogonal finite-difference time-domain method," Phys. Rev. B, Condens. Matter, vol. 58, pp. 7252-7259, 1998.

[11] A. J. Ward and J. B. Pendry, "A program for calculating photonic band structures, Green's functions and transmission/reflection coefficients using non-orthogonal FDTD method," Comput. Phys. Commun., vol. 128 , pp. 590-621, 2000.

[12] A. Nicolet, F. Zolla, and S. Guenneau, "Modelling of twisted optical waveguides with edge elements," Eur. Phys. J. Appl. Phys., vol. 28, pp. 153-157, 2004.

[13] J. Chandezon, G. Raoult, and D. Maystre, "A new theoretical method for diffraction gratings and its numerical application," J. Opt., vol. 11, pp. 235-241, 1980.
[14] D. M. Shyroki, "Squeezing of open boundaries by Maxwell-consistent real coordinate transformation," IEEE Microw. Wireless Compon. Lett., vol. 16, no. 11, pp. 576-578, Nov. 2006.

[15] Y. Kobayashi and T. Senju, "Resonant modes in shielded uniaxial anisotropic dielectric rod resonators," IEEE Trans. Microw. Theory Tech., vol. 41, no. 10, pp. 2198-2205, Oct. 1993.

[16] J.-M. Guan and C.-C. Su, "Resonant frequencies and field distributions for the shielded uniaxially anisotropic dielectric resonator by the FD-SIC method," IEEE Trans. Microw. Theory Tech., vol. 45, no. 10, pp. 1767-1777, Oct. 1997.

[17] M. Gastine, L. Courtols, and J. L. Dormann, "Electromagnetic resonances of free dielectric spheres," IEEE Trans. Microw. Theory Tech., vol. MTT-15, no. 12, pp. 694-700, Dec. 1967.

[18] A. Julien and P. Guillon, "Electromagnetic analysis of spherical dielectric shielded resonators," IEEE Trans. Microw. Theory Tech., vol. MTT-34, no. 6, pp. 723-729, Jun. 1986.

[19] E. Madelung, Die Mathematischen Hilfsmittel des Physikers. Berlin, Germany: Springer, 1957.

[20] F. L. Teixeira and W. C. Chew, "Systematic derivation of anisotropic PML absorbing media in cylindrical and spherical coordinates," IEEE Microw. Guided Wave Lett., vol. 7, no. 11, pp. 371-373, Nov. 1997.

[21] P. G. Petropoulos, "Reflectionless sponge layers for the numerical solution of Maxwell's equations in cylindrical and spherical coordinates," Appl. Numer. Math., vol. 33, pp. 517-524, 2000.

[22] R. B. Lehoucq, D. C. Sorensen, and C. Yang, ARPACK Users' Guide: Solution of Large-Scale Eigenvalue Problems With Implicitly Restarted Arnoldi Methods. Philadelphia, PA: SIAM, 1998.

[23] J. D. Jackson, Classical Electrodynamics, 3rd ed. New York: Wiley, 1998 , ch. 8.

[24] R. Holland, "Pitfalls of straircase meshing," IEEE Trans. Electromagn. Compat., vol. 35, no. 4, pp. 434-439, Nov. 1993.

[25] S. Dey and R. Mittra, "A conformal finite-difference time-domain technique for modeling cylindrical dielectric resonators," IEEE Trans. Microw. Theory Tech., vol. 47, no. 9, pp. 1737-1739, Sep. 1999.

[26] M. Born and E. Wolf, Principles of Optics. Oxford, U.K.: Pergamon, 1968.

[27] A. V. Lavrinenko and V. V. Zhilko, "Application of coordinate-free effective medium theory to periodically plane-stratified anisotropic media," Microw. Opt. Technol. Lett., vol. 15, pp. 54-57, 1997.

[28] R. D. Meede, A. M. Rappe, K. D. Brommer, J. D. Joannopoulos, and O. L. Alerhand, "Accurate theoretical analysis of photonic bandgap materials," Phys. Rev. B, Condens. Matter, vol. 48, pp. 8434-8437, 1993.

[29] R. D. Meede, A. M. Rappe, K. D. Brommer, J. D. Joannopoulos, and O. L. Alerhand, "Erratum to "Accurate theoretical analysis of photonic bandgap materials'," Phys. Rev. B, Condens. Matter, vol. 55, p. 15942 , 2000.

[30] C.-P. Yu and H. C. Chang, "Compact finite-difference frequency-domain method for the analysis of two-dimensional photonic crystals," Opt. Express, vol. 12, pp. 1397-1408, 2004.

[31] J. Riishede, "Modelling photonic crystal fibres with the finite difference method," Ph.D. dissertation, Commun., Opt., Mater., Tech. Univ. Denmark, Lyngby, Denmark, 2005.

[32] S. G. Johnson and J. D. Joannopoulos, "Block-iterative frequency-domain methods for Maxwell's equations in a planewave basis," Opt. Express, vol. 8, pp. 173-190, 2001.

[33] H. C. van de Hulst, Light Scattering by Small Particles. New York: Wiley, 1957, sec. 10.5.

[34] A. Ivinskaya, Oct. 2006, private communication.

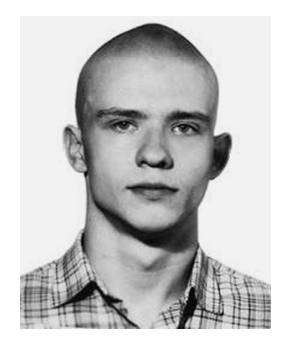

Dzmitry M. Shyroki was born in Minsk, Belarus, on September 24, 1981. He received the Specialist degree from Belarusian State University, Minsk, Belarus, in 2004, and is currently working toward the Ph.D. degree at the Technical University of Denmark, Lyngby, Denmark. 